\title{
Use of chromosome microdissection in fish molecular cytogenetics
}

\author{
Frederico Henning ${ }^{1}$, Vladimir Trifonov ${ }^{2,3}$ and Lurdes Foresti de Almeida-Toledo ${ }^{1}$ \\ ${ }^{I}$ Departamento de Genética e Biologia Evolutiva, Universidade de São Paulo, São Paulo, SP, Brazil. \\ ${ }^{2}$ Institute of Cytology and Genetics, Siberian Branch of the Russian Academy of Sciences, \\ Novosibirsk, Russia. \\ ${ }^{3}$ Centre for Veterinary Science, University of Cambridge, Cambridgeshire, UK
}

\begin{abstract}
Chromosome microdissection is a technique in which whole chromosomes or chromosomal segments are dissected under an inverted microscope yielding chromosome-specific sequences. Several protocol modifications introduced during the past 15 years reduced the number of chromosomes required for most applications. This is of particular interest to fish molecular cytogenetics, since most species present highly uniform karyotypes which make impossible the collection of multiple copies of the same chromosome. Probes developed in this manner can be used to investigate chromosome homologies in closely related species. Here we describe a protocol recently used in the gymnotiform species group Eigenmannia and review the major steps involved in the generation of these markers focusing on protocol modifications aiming to reduce the number of required chromosomes.
\end{abstract}

Key words: fluorescence in situ hybridization, chromosome painting, sex chromosomes, cross-FISH.

Received: August 21, 2006; Accepted: April 18, 2007.

Chromosome-specific sequences are highly desired in studies focusing on comparative genomics and genomic organization. The two most straightforward paths for obtaining chromosome-specific makers are flow-sorting, commonly referred to as FACs (fluorescence activated cell sorting), and chromosome microdissection. In flowsorting, chromosomes are sorted using a laser system that distinguishes chromosomes depending on their size and fluorochrome affinity (AT, GC base content) while in chromosome microdissection chromosomes or chromosomal segments are literally scraped and collected.

It is generally assumed that flow-sorting generates paints of greater complexity and coverage because this method allows the collection of massive amounts of chromosomes (around 300-500) in a highly automated procedure (Ferguson-Smith et al., 1998). However, the costs of equipment and need of high quality cell cultures make this technique not suitable for most fish cytogenetics laboratories. Specifically concerning genetic studies in fish, a new difficulty arises, since most fish species present a highly uniform karyotype regarding chromosomal size and base content.

Chromosome microdissection was originally developed in studies involving Drosophila polytene chromo-

Send correspondence to Frederico Henning. Departamento de Genética e Biologia Evolutiva, Universidade de São Paulo, Rua do Matão 277, Sala 237, 05508-090 São Paulo, SP, Brazil. E-mail: henning@ib.usp.br. somes as a way to obtain DNA markers from specific chromosomal regions and was quickly applied to mammalian genomes during the 80s (Fan, 2002). The subsequent development of in situ hybridization procedures and establishment of DOP-PCR (Telenius et al., 1992) allowed the application of chromosomal probes obtained by microdissection in FISH (fluorescence in situ hybridization) procedures (sometimes referred to as microFISH). This technique has been applied in several studies involving fish species, and has been particularly useful in studies focused on sex-chromosomes (Reed et al., 1995; Harvey et al., 2002; Liu et al., 2002).

Microdissection is commonly regarded as a labor intensive method since most protocols require the collection of 20 chromosomal copies. However, protocol modifications have been made in order to reduce the number of chromosomes (one to five chromosomes are sufficient depending on the application), turning microdissection into a routine procedure whilst maintaining probe quality (Guan et al., 1994; Engelen et al., 1998; Christian et al., 1999; Weimer et al., 1999).

Amplification of the chromosomal libraries is done by degenerate oligonucleotide primed PCR (DOP-PCR) (Telenius et al., 1992). A pre-amplification step (eight cycles) has been found to increase probe coverage (Guan et al., 1994). Pre-amplification involves the use of alternative DNA polymerases, particularly T7 DNA polymerase (commercially available as Sequenase) due to its higher pro- 
cessivity, particularly in the 25 to $35^{\circ} \mathrm{C}$ range, which are the temperatures used in primer annealing and extension. Since Sequenase is not thermo-stable, small quantities $(0.3 \mathrm{U})$ must be added at each of the eight cycles during annealing. The use of Thermosequenase has also been reported (Christian et al., 1999). Since this enyme is thermostable, its use minimizes risk of contamination involved in adding additional enzyme each cycle. It should be noted however, that Thermosequenase is a modified Taq polymerase, and therefore presents highest processivity at elevated temperatures. Therefore, the use of temperature ramps between annealing and extension is recommended. The final 20 cycles (high-stringency cycles) can be performed using regular Taq polymerase, since incorporation of the 5 ' non-degenerate sequence of the primer insures specific amplification at high extension temperatures. (Guan et al., 1994)

Here we describe a protocol, slightly modified from Weimer et al. (1999), recently used to microdissect and amplify autosome and X-linked sequences of Eigenmannia virescens. Samples used for microdissection and FISH present $2 \mathrm{n}=38 \mathrm{XY}$ and $31 / 32$, as previously described by Almeida-Toledo et al. (2001). Direct kidney preparations was obtained from individuals collected at small tributaries of the Tietê and Parapanema river basins (Brazil) in 2006, and have been stored in fixative at $-20{ }^{\circ} \mathrm{C}$.

Suspensions were dropped on coverslips and subjected to regular Giemsa staining. The use of coverslips is necessary since the objectives and material are positioned underneath and on top of the coverslip, respectively, in an inverted microscope. Slide thickness impairs such cross focusing with $40 \mathrm{x}$ and $100 \mathrm{x}$ objectives. Cell suspensions should be diluted to ensure good distribution of metaphases, and special attention should be given to spreading and pre-treatment. Usually, fresh suspensions can be used without any treatment. (Henegariu et al., 2001).

The needles were prepared from borosilicate rods (Harvard Apparatus - Massachusetts, USA) using a twostep pipette puller (Narishige, Tokyo, Japan). Glass capillaries can also be used, although they are less stable and more susceptible to breakage. Needles were loaded to a micromanipulator attached to the microscope. Siliconized pipettes were also prepared with the pipette puller or by manual pulling after heat exposure, and the tip was gently broken in order to create a small opening where the chromosomes and collection solution should are stored during microdissection. The pipette was fixed to the microscope table using commercially available modeling clays. All equipment was exposed to UV light prior to use.

Centralizing the needle and pipette before loading the coverslip has been found to considerably facilitate procedures. While manipulating the coverslip and microscope table, it is recommended to maintain the needle at the high position to avoid breakage. Detachment of the chromosomes from the coverslip was achieved by gently scraping the chromosome along its vertical axis, causing the chromosome to fold around itself and finally bind to the needle.

Chromosomes were transferred to the pipette containing a collection solution $(10 \mathrm{mM} \mathrm{NaCl}, 10 \mathrm{mM}$ Tris- $\mathrm{HCl}$ pH 7.5, 1 mM EDTA pH 7.5-8.0, 0.1\% SDS, 0.1\% Triton $\mathrm{X} 100,500 \mu \mathrm{M}$ proteinase $\mathrm{K}$, and $30 \%$ glycerol). The collection solution was added by capillarity to the pipette prior to use. The use of topoisomerase instead of proteinase has also been reported (Guan, 2002).

The chromosomes were transferred by inserting the tip of the needle into the pipette containing the collection solution. The same needle was used to pick up further chromosomes. The pipette containing the chromosomes was incubated in a humid chamber at $60{ }^{\circ} \mathrm{C}$ for one hour. Incubation in a humid chamber and the use of glycerol are both effective in controlling evaporation. The chromosomes were then transferred to the pre-amplification mixture described bellow by breaking the tip of the pipette inside the microtube containing the PCR solution.

The pre-amplification PCR mix used consisted of $24 \mathrm{mM}$ Tris- $\mathrm{pH} 7.5,12 \mathrm{mM} \mathrm{MgCl} 2,30 \mathrm{mmol} / \mathrm{L} \mathrm{NaCl}(0.6 \mathrm{x}$ Reaction Buffer), 0.2 mM dNTP's, $5 \mu \mathrm{M}$ 6-MW primer (5' CCG ACT CGA GNN NNN NAT GTG G 3'), in a $5 \mu \mathrm{L}$ final volume. The reaction profile used is $90{ }^{\circ} \mathrm{C}$ for $1 \mathrm{~min}$, $25{ }^{\circ} \mathrm{C}$ for $2 \mathrm{~min}$, and $4{ }^{\circ} \mathrm{C}$ for $2 \mathrm{~min}$. As mentioned before, $0.3 \mathrm{U}$ of Sequenase should be added at each cycle during annealing. A first step of denaturation $\left(5 \mathrm{~min}\right.$ at $\left.90{ }^{\circ} \mathrm{C}\right)$ is necessary to inactivate proteinase activity in the collection solution.

For the standard DOP amplification after the preamplification step ( 30 cycles), $50 \mu \mathrm{L}$ of the following mix is added to the previous PCR product: $10 \mu \mathrm{L}$ of $10 \mathrm{x} \mathrm{NH}_{4}$ Reaction Buffer $\left(160 \mathrm{mM}\left(\mathrm{NH}_{4}\right)_{2} \mathrm{SO}_{4}, 670 \mathrm{mM}\right.$ Tris-HCl, $0.1 \%$ Tween-20), $0.2 \mathrm{mM}$ dNTP's, $5 \mu \mathrm{M}$ 6-MW primer and $0.1 \mathrm{U}$ Taq polymerase. The high-temperature reaction profile is $92^{\circ} \mathrm{C}$ for $1 \mathrm{~min}, 56^{\circ} \mathrm{C}$ for $2 \mathrm{~min}$, and $70{ }^{\circ} \mathrm{C}$ for $2 \mathrm{~min}$. A final 5 min extension step at $72{ }^{\circ} \mathrm{C}$ should be added.

The probe was PCR labeled with Biotin-16-dUTP (Roche) in 28 DOP-PCR cycles using as template DNAdeletar $1 \mu \mathrm{L}$ of the primary DOP-PCR product as template DNA. Control of non-specific signals was achieved using salmon sperm DNA $(15 \mathrm{mg})$ and a pre-hybridization step (15-30 min at $\left.37^{\circ} \mathrm{C}\right)$.

Figure 1 shows hybridization of a probe developed from a single microdissected autosome of Eigenmannia sp. 2 . Note that the probe distinctively hybridizes to the entire chromosome pair. Figure 2 shows hybridization of X-heterochromatin and $\mathrm{Y}$-specific probes obtained by microdissection (three and four copies of each chromosome, respectively) from E. virescens and Eigenmannia sp. 2, respectively. The probe derived from the heterochromatic segment of $E$. virescens $\mathrm{X}$ chromosome hybridizes successfully to the $\mathrm{X}$ specific heterochromatin as shown in Figure $2 \mathrm{~A}$. 


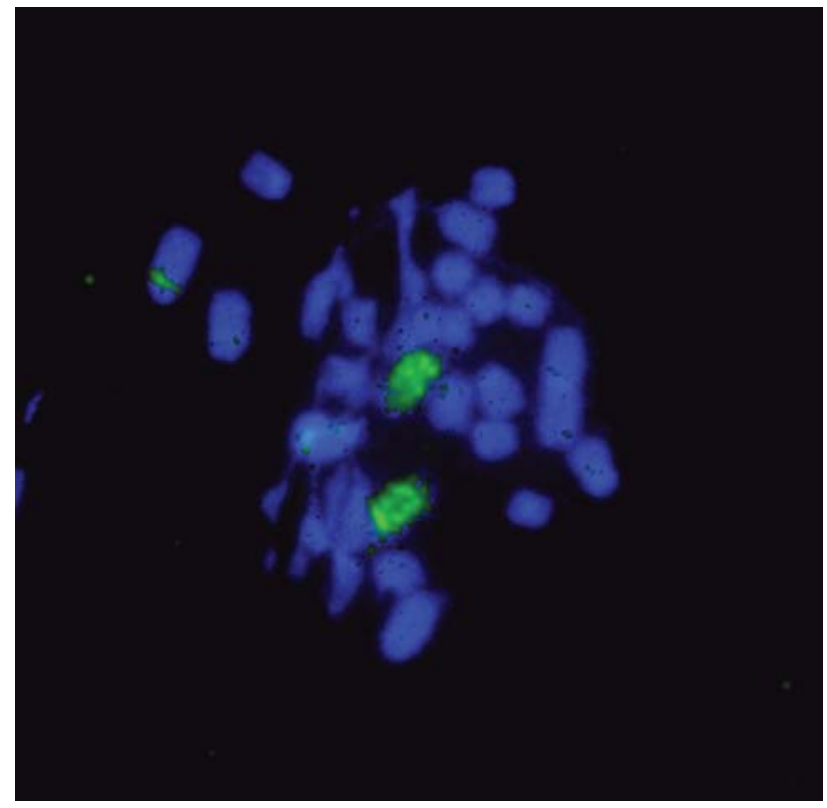

Figure 1 - Hybridization of a probe developed from a single microdissected autosome of Eigenmannia sp. 2.
Furthermore, DAPI (4'-6-Diamidino-2-phenylindole) counterstain on E. virescens (Figure 2B) revealed a distinct striped pattern of $\mathrm{X}$ heterochromatin, where ATrich sequences seem to be alternated with GC-rich sequences. The $\mathrm{Y}$-derived probe hybridizes to the $\mathrm{Y}, \mathrm{X}_{1}$, and $\mathrm{X}_{2}$ chromosomes (Figure 2C). Hybridizations to $\mathrm{X}$ chromosomes, as seen in Figure 2C, were not total, adding further evidence to the presumable a loss of repetitive sequences during the fusion event that originated the $\mathrm{Y}$ chromosome (Almeida-Toledo et al., 2000).

Recent modifications from the original protocols have made the microdissection procedure applicable for molecular-cytogenetics and comparative genomic research. Probes developed using microdissection have been reported to show properties similar to those obtained by flow-sorting (Griffin et al., 1999), although this may not hold in cross-FISH experiments with distant species microdissection is a suitable procedure for comparison of closely related species groups. Probes obtained using this protocol have successfully revealed chromosome homologies in

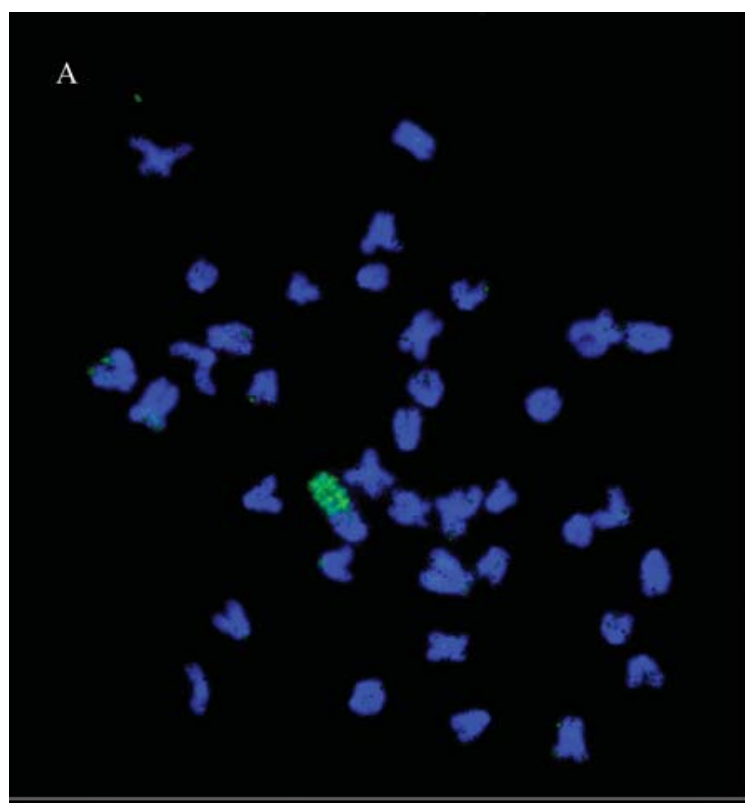

B
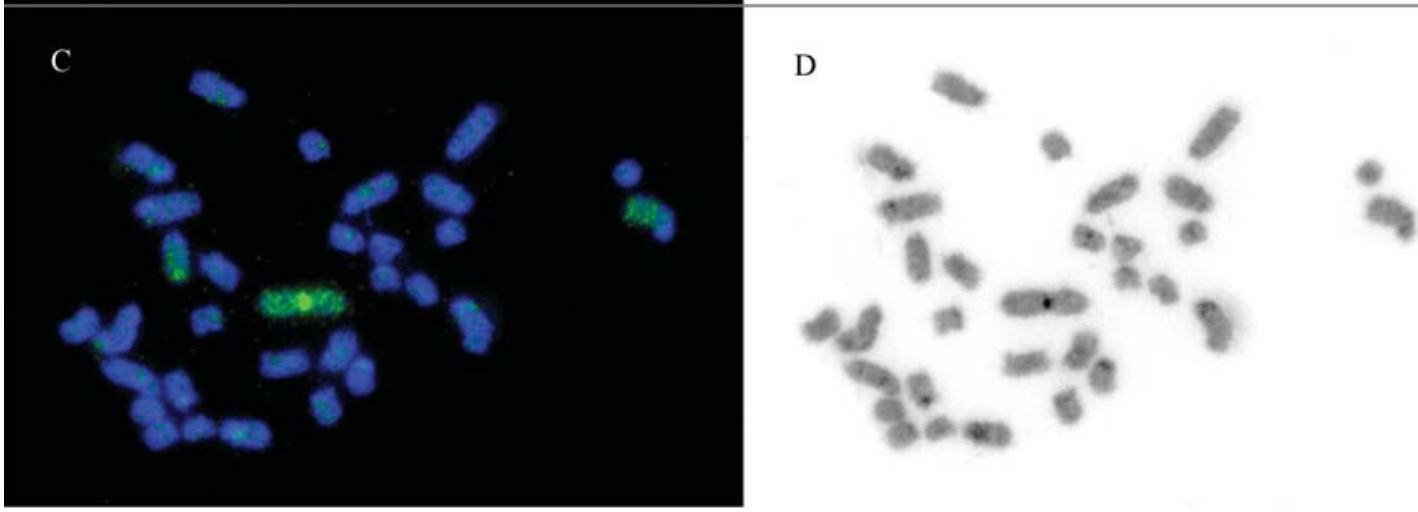

Figure 2 - Hybridization patterns using A) a probe developed from three copies of X chromosome heterochromatin of Eigenmannia virescens; B) DAPI counterstain of A; C) a probe derived from Eigenmannia sp. 2 Y chromosome; D) DAPI counterstain of C. 
Eigenmannia species (Henning F, Trifonov V, FergusonSmith MA and Almeida-Toledo, unpublished data).

An interesting application of microdissection is the construction of plasmid library from the DOP-amplified fragments. DOP-PCR fragments are, however, considerably small (200-500 bp) and fragmented (the 5' sequence of the 6-MW primer is expected to occur at each $4 \mathrm{~kb}$ ). These libraries can however, be useful for isolation and sequencing of repetitive DNA using a combination of FISH and blotting techniques (Southern, dot or slot blotting) for screening (Shibata et al., 1999; Madalena and Gorab, 2005) or as probes for retrieving region specific sequences from a BAC library. This procedure is highly desired in studies focused on B-chromosome investigations, since sequence data can help assessing the evolutionary origins of supernumerary chromosomes (Bugrov et al., 2004, Rubstov et al., 2004) and is of potential use in fish breeding (Yi et al., 2003).

Despite being a numerous and highly diverse group, chromosome painting has been applied to only a handful of species (Phillips et al., 2001; Harvey et al., 2002; Liu et al., 2002). The possibility of developing paints from few, or even single microdissected chromosomes is promising, considering common characteristics of fish chromosomes such as uniformity in size and morphology.

\section{Acknowledgments}

We thank the anonymous reviewers for the helpful suggestions and Dr. Eduardo Gorab (University of São Paulo) and Dr. Malcolm Ferguson-Smith (University of Cambridge) for permission to use facilities and equipment. This work was supported by CNPq and FAPESP.

\section{References}

Almeida-Toledo LF, Foresti F, Daniel-Silva MF and Toledo Filho AS (2000) Sex chromosome evolution in fish: The formation of the neo-Y chromosome in Eigenmannia (Gymnotiformes). Chromosoma 109:197-200.

Almeida-Toledo LF, Foresti F, Pequignot EV and Daniel-Silva MF (2001) XX:XY sex chromosome system with X heterochromatinization: An early stage of sex chromosome differentiation in the Neotropic electric eel Eigenmannia virescens. Cytogenet Cell Genet 95:73-78.

Bugrov AG, Karamysheva TV, Rubtsov DN, Andreenkova OV and Rubtsov NB. (2004) Comparative FISH analysis of distribution of B chromosome repetitive DNA in A and B chromosomes in two subspecies of Podisma sapporensis (Orthoptera, Acrididae). Cytogenet Genome Res 106:284288.

Christian AT, Garcia HE and Tucker JD (1999) PCR in situ followed by microdissection allows whole chromosome painting probes to be made from single microdissected chromosomes. Mamm Genome 10:628-631.
Engelen JJ, Albrechts JC, Hamers GJ and Geraedts JP (1998) A simple and efficient method for microdissection and microFISH. J Med Genet 35:265-268.

Fan YS (2002) Molecular Cytogenetics: Protocols and Applications. Humana Press, Totowa, 411 pp.

Ferguson-Smith MA, Yang F and O'Brien PC (1998) Comparative mapping using chromosome sorting and painting. Ilar J 39:68-76.

Garcia C, Moreira-Filho O, Bertollo LAC and Centofante L (2003) B chromosomes and natural triploidy in Rhamdia sp. (Pisces, Siluriformes, Heptapteridae). Cytologia 68:403411.

Griffin DK, Haberman F, Masabanda J, O'Brien P, Bagga M, Sazanov A, Smith J, Burt DW, Ferguson-Smith MA and Wienberg J (1999) Micro- and macrochromosome paints generated by flow cytometry and microdissection: Tools for mapping the chicken genome. Cytogenet Cell Genet $87: 278-281$.

Guan X-Y (2002) Chromosome microdissection. In: Fan Y-S (ed) Molecular Cytogenetics: Protocols and Applications. Humana Press, New Jersey, pp 67-76.

Guan X-Y, Meltzer PS and Trent JM (1994) Rapid generation of whole chromosome painting probes (WCPs) by chromosome microdissection. Genomics 22:101-107.

Harvey SC, Masabanda J, Carrasco LA, Bromage NR, Penman DJ and Griffin DK (2002) Molecular-cytogenetic analysis reveals sequence differences between the sex chromosomes of Oreochromis niloticus: Evidence for an early stage of sexchromosome differentiation. Cytogenet Genome Res 97:76-80.

Henegariu O, Heerema NA, Lowe Wright L, Bray-Ward P, Ward DC and Vance GH (2001) Improvements in cytogenetic slide preparation: Controlled chromosome spreading, chemical aging and gradual denaturing. Cytometry 43:101-109.

Liu JD, Yi MS, Zhao G, Zhou F, Wang DQ and Yu QX (2002) Sex chromosomes in the spiny eel (Mastacembelus aculeatus) revealed by mitotic and meiotic analysis. Cytogenet Genome Res 98:291-297.

Madalena CR and Gorab E (2005) A chromosome end satellite of Rhynchosciara americana (Diptera, Sciaridae) resembling nematoceran telomeric repeats. Insect Mol Biol 14:255-262.

Moreira-Filho O, Galetti PM and Bertollo LA (2004) B chromosomes in the fish Astyanax scabripinnis (Characidae, Tetragonopterinae): An overview in natural populations. Cytogenet Genome Res 106:230-234.

Phillips RB, Konkol NR, Reed KM and Stein JD (2001) Chromosome painting supports lack of homology among sex chromosomes in Oncorhynchus salmo and Salvelinus (Salmonidae). Genetica 111:119-123.

Reed KM, Bohlander SK and Phillips RB (1995) Microdissection of the $\mathrm{Y}$ chromosome and fluorescence in situ hybridization analysis of the sex chromosomes of lake trout, Salvelinus namaycush. Chromosome Res 3:221-226.

Rubtsov NB, Karamysheva TV, Andreenkova OV, Bochkaerev MN, Kartavtseva IV, Roslik GV and Borissov YM (2004) Comparative analysis of micro and macro B chromosomes in the Korean field mouse Apodemus peninsulae (Rodentia, 
Murinae) performed by chromosome microdissection and FISH. Cytogenet Genome Res 106:289-294.

Shibata F, Hizume M and Kuroki Y (1999) Chromosome painting of Y chromosomes and isolation of a Y chromosome-specific repetitive sequence in the dioecious plant Rumex acetosa. Chromosoma 108:266-270.

Telenius H, Carter NP, Bebb CE, Nordenskjold M, Ponder BA and Tunnacliffe A (1992) Degenerate oligonucleotideprimed PCR: General amplification of target DNA by a single degenerate primer. Genomics 13:718-725.
Weimer J, Kiechle M, Senger G, Wiedemann U, Ovens-Raeder A, Schuierer S, Kautza M, Siebert R and Arnold N (1999) An easy and reliable procedure of microdissection technique for the analysis of chromosomal breakpoints and marker chromosomes. Chromosome Res 7:355-362.

Yi MS, Li YQ, Liu JD, Zhou L, Yu QX and Gui JF (2003) Molecular cytogenetic detection of paternal chromosome fragments in allogynogenetic gibel carp, Carassius auratus gibelio Bloch. Chromosome Res 11:665-671.

Associate Editor: Luiz Antonio Carlos Bertollo

License information: This is an open-access article distributed under the terms of the Creative Commons Attribution License, which permits unrestricted use, distribution, and reproduction in any medium, provided the original work is properly cited. 